\title{
NOUVELLES ESPÈCES DU GENRE CYCLOPOSTHIUM HABITANT L'INTESTIN DU CHEVAL
}

\author{
Par A. STRELKOW
}

Au cours de mes recherches sur la morphologie et la cytologie des Cycloposthiidx de l'intestin du cheval, j'ai rencontré plusieurs formes du genre Cycloposthium Bundle, inconnues jusqu'ici et dont je donnerai une courte description.

Le matériel que j'ai étudié provient de l'abattoir de Leningrad. Grâce à l'amabilité du docteur Wolferts auquel j'exprime toute ma reconnaissance, j'ai réussi à étudier les échantillons in vivo sur une table chauffante. Les infusoires de l'intestin du cheval, comme l'avait déjà signalé Bundle (1895), sont très sensibles à un abaissement de température ; sous l'action du froid, ils deviennent immobiles et invaginent leur péristome. Pour éviter cet inconvénient, je me suis servi de boîtes de Petri réchauffées et de fixateurs chauffés ; malgré ces précautions, j'ai rarement réussi à obtenir de gros Cycloposthium fixés avec le ciliophore épanoui.

Les espèces nouvelles ont été étudiées in viøo et sur des préparations fixées par le liquide de Schaudinn, par le Zenker-formol ou par les mélanges osmiques et colorées in toto. Les infusoires étaient colorés à l'hématoxyline ferrique de Heidenhain ou à l'hématoxyline Delafield.

Les mensurations ont été faites sur les individus neutres dans le sens de Dogiel (1925), e'est-à-dire ne se trouvant à aucun stade du processus de division.

Nous trouvons la première description exacte de l'Entodinium bipalmatum de l'intestin du cheval chez Fiorentini(1) (1890). Bundle (1895) (2) a placé cette espèce dans un genre spécial, le genre Cycloposthium. Günther (1900) (3) donne des détails sur la morphologie du C. bipalmatum. Da Cunha (1915) (4) décrit trois nouvelles espèces de cæcum du cabiai (Hydrochœrus capibara L.), C. hydro-

(1) Fionentrni. - Intorno ai Protisti dell intestino degli equini. Pavia, 1890.

(2) BUNdLe (A.). - Zeitschr. t. wissensch. Zoologie, Lx, 1895, p. 284-350.

(3) GüNTHER. - Zeitschr. f. wissenschaft. Zoologie, LXVII, 1900, p. 640-662.

(4) Da Cunha. - Mem. Inst. Osw. Cruz, VII, 1915, p. 139-145.

Annales de Parasitologie, T. VI, $\mathrm{N}^{\circ} 2 .-1^{\mathrm{er}}$ avril 1928 , p. 164-178. 
chori, C. incurvum, C. compressum. Gassovsky (1918) (1) décrit C. dentiferum et C. ischiscawai du cæcum du cheval. Enfin Dogiel, 1923 (2) eł 1925 (3), décrit en détail le processus de conjugaison des Cycloposthium, puis en 1925 (4), il étudie la nutrition des Cycloposthiidæ.

Dans la description des espèces nouvelles qui va suivre, je considère comme face dorsale celle où se trouve le macronucléus et comme face ventrale celle sur laquelle se rétracte le péristome pendant son invagination. Je nomme " queue " la partie du corps qui se trouve derrière les faisceaux caudaux.

Au cours des mensurations, j'ai pris pour largeur le diamètre du corps à peu près en son milieu dans la direction dorso-ventrale et pour épaisseur le diamètre latéral d'un côté à l'autre.

\section{Genre Cycloposthium Bundle, 1895}

Le corps a une forme stable, qui dépend de la présence d'un squelette externe en forme de cuirasse. Ce squelette était considéré par les auteurs précédents comme une couche alvéolaire, logée immédiatement sous la cuticule. Les alvéoles de cette couche représentent les alvéoles du squelette qui sont visibles à travers la cuticule et forment à la surface du corps un dessin polygonal, très évident chez toutes les espèces ci-dessous mentionnées.

Le corps est dénué de cils, il n’y a des membranelles que sur le péristome et dans les deux faisceaux postérieurs, les " caudalia " de Bundle. Ces derniers sont disposés sur les faces ventrale et dorsale; le faisceau dorsal est logé un pẻu plus en arrière que le faisceau ventral.

Le pharynx présente des fibrilles en forme de rubans, logées dans sa paroi et se rétrécit après avoir quitté le péristome. En s'élargissant plus en arrière, il porte une fente longitudinale à gauche, de sorte que la cavité du pharynx communique avec l'endoplasme. Les fibrilles pharyngiennes se prolongent jusqu'à l'extrémité postérieure du corps, le long du côté droit et de la partie dorsale du pharynx.

Il existe un tube anal situé sur la face ventrale, prenant naissance dans l'endoplasme et s'ouvrant par l'orifice anal immédiatement au delà de la base du faisceau caudal ventral.

(1) Gassovsky. - Travaux de la Société des Naturalistes de Petrograd. Section de Zoologie et de Physiol., XLIX, 1918, p. 20-36.

(2) DoGisL (V.). The transformation of the male pronucleus in a spermatozoon in certain Infusoria during their conjugation. Petrograd, 1923.

(3) Dogres (V.). - Arch. für Protistenk., L, 1925, p. 283-442.

(4) Dogrel (V.). - Travaux de la Société des Naturalistes de Leningrad. Section de Zoologie et Physiol., LIV, 1925, p. 69-93. 
Le macronucleus, de forme allongée, est logé le long de la paroi dorsale du corps ; un petit micronucleus, de forme ellipsoïdale, est logé dans l'échancrure du macronucleus.

A côté du macronucleus se trouve une rangée longitudinale de vacuoles contractiles en nombre constant pour chacune des espèces décrites. En ce point, passe le ruban squelettique ("Leiste ", d'après Bundle).

- Les différentes espèces de Cycloposthium ont été découvertes dans le cæcum du cheval et dans celui du cabiai (Hydrochœrus capibara L.).

Etant donné que les nouvelles espèces sont conformées sur le même plan que celle que l'on rencontre communément dans le cæcum du cheval, Cycloposthium bipalmatum, je donne tout d'abord une courte description de cette espèce.

\section{Cycloposthium bipalmatum (Fiorentini, 1890)}

Le corps, de forme constante, est aplati des deux côtés et un peu aminci en arrière (fig. 1). Derrière les «caudalia », le corps se transforme en une queue très courte et arrondie. Le squelette ne recouvre pas la queue; il n'y est représenté que sous la forme de deux lobes latéraux. Au pôle antérieur du corps, se trouve le péristome, capable de s'invaginer et composé des différentes parties décrites par Bundle (1895). Le pharynx, au delà du péristome, présente, en arrière de sa portion dilatée, au niveau de l'extrémité antérieure du macronucleus, une fente sur sa paroi gauche et les fibrilles pharyngiennes longent son côté droit et la face dorsale du corps. Sur le pôle postérieur, les fibrilles atteignent le niveau des " caudalia ". Le tube anal est situé sur la face ventrale et s'ouvre par l'orifice anal immédiatement derrière la base du faisceau caudal ventral. Des fibrilles en forme d'anneau sont logées dans les parois du tube.

Le macronucleus, de forme allongée, est situé dans l'ectoplasme sur le côté dorsal; son extrémité antérieure se recourbe vers la paroi ventrale, s'inclinant à droite; le micronucleus est logé dans l'échancrure du macronucleus à peu près au milieu du côté dorsai de ce dernier. Les vacuoles contractiles, au nombre de 4 à 5 , forment une rangée longitudinale près du macronucleus. Vues de côté, les vacuoles sont souvent cachées par le macronucleus adjacent. C'est aussi à côté du macronucleus que se trouve le ruban squelettique longitudinal (" Leiste " de Bundle).

La longueur du corps varie entre 65 et $115 \mu$ (moyenne $89 \mu$ ); la largeur varie entre 26 et $49 \mu$ (moyenne $38 \mu$ ); la longueur du macronucleus est de 43 à $73 \mu$ (moyenne $59 \mu$ ). 
La différence entre les dimensions données ici et celles qu'a données Bundle me semble provenir de ce que la longueur maxima donnée par Bundle $(191 \mu)$ se rapporte probablement à un exemplaire en voie de division. Quant à moi, ainsi que je l'ai indiqué précédemment, je n'ai pris en considération que les exemplaires à l'état de repos.

Les populations d'infusoires provenant des intestins de différents

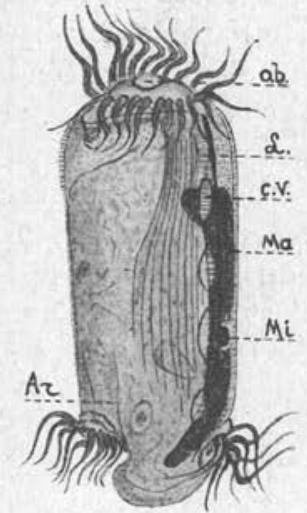

A.

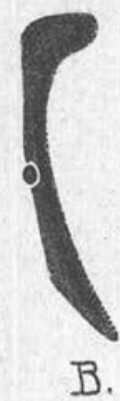

B.

Fig. 1. - Cycloposthium bipalmatum. A, vu du côtê gauche ;

B, macronucléus vu du côté dorsal ; $\times 280$ (1).

chevaux peuvent être de races particulières de $C$. bipalmatum, caractérisées par leurs dimensions. Ainsi dans les deux cas suivants l'analyse biométrique nous montre une différence très nette: Dans une population, la longueur du corps varie entre 65 et $100 \mu$, $\mathrm{M}=82,1 \pm 1,2 \mu, \mathrm{C}=9,00 / 0, n=50$; dans une autre, la longueur du corps varie entre 88 et $115 \mu, \mathrm{M}=100,1 \pm 0,9 \mu, \mathrm{C}=6,60 / 0$, $n=50$. La différence des moyennes est tellement plus grande que l'erreur moyenne de cette différence qu'elle permet de conclure à deux races différentes :

$$
\left(\mathrm{M}_{1}-\mathrm{M}\right) \pm \sqrt{m_{1}^{2}+m^{2}}=18,0 \pm 1,5 .
$$

(1) LÉgende GÉNÉrale des figures. - A $r$, tube rectal ; $\mathrm{B}$, bouclier ; $\mathrm{B} d$, bord dorsal du bouclier; $\mathrm{B} v$, bord ventral du bouclier; Can, canalicule faisant communiquer la vacuole contractile avec l'extérieur; $c r$, crête longitudinale sur la côte dorsale; $c v$, vacuoles contractiles; $d$, dent; $e c$, ectoplasme ; $e n$, endoplasme ; Eps, extrémité postérieure du squelette dans la queue ; Fc, fibrilles partant de la base des " caudalia »; Fph, fibrilles pharyngiennes; $g$, gouttière entre le lobe dorsal du bouclier et la crête longitudinale du côté dorsal; L, ruban squelettique ( $\propto$ Leiste $»$ de Bundle); $\mathrm{M} a$, macronucléus ; $\mathrm{M} i$, micronucléus; $\mathrm{O} b$, bouche ; $p h$, pharynx; $q$, queue; Rc, retractores ciliophori ; Sk, squelette. 
En décrivant cette espèce en 1918, Gassovsky donne des caractères qui paraissent être une combinaison de caractères du Cycloposthium dentiferum et d'une seconde espèce, décrite par moi ci-dessous. Les deux espèces se rencontrant souvent ensemble dans une même population, il est possible que Gassovsky les ait confondues, surtout lorsqu'il existe de nombreux stades de division, ce qui rend la différence entre les deux espèces plus délicate.

Le corps du $C$. dentiferum, comparable à un parallélépipède, est comprimé des deux côtés. S'amincissant particulièrement au delà des " caudalia ", il forme une excroissance caudiforme. Cette excroissance est droite, s'élargit un peu vers son extrémité postérieure, mais ne forme pas de crochet ou de fer à cheval, comme l'indique Gassovsky pour le C. dentiferum. La forme permanente du corps dépend de la présence du squelette extérieur, qui ne laisse libre que la queue. Celle-ci n'est que partiellement protégée par deux lobes latéraux de la cuirasse (fig. 2 A., $E$. p. s.). C'est pourquoi la queue peut changer de forme quand l'animal se gorge de gros morceaux de nourriture.

Cefte espèce a été dénommée dentiferum à cause d'une excroissance dentelée située sur le côté ventral de son extrémité antérieure (fig. 2 A. et $3, d$ ). Cette excroissance est formée par le bord antérieur de la cuirasse, dont les parois latérales s'unissent sur le côté ventral et $\mathrm{y}$ font une saillie en avant. La dent ainsi formée ressort plus du côté gauche, grâce à une petite échancrure du bord antérieur de la cuirasse à la base de la dent. Du côté droit, la dent semble être moins développée que du côté gauche; et il n'y a pas de pareille échancrure sur la paroi droite (fig. 3).

Le péristome, situé à l'extrémité antérieure du corps est capable de s'invaginer comme chez toutes les espèces de Cycloposthium. Les parties décrites par Bundle chez le $C$. bipalmatum existent aussi chez le $C$. dentiferum.

Un large pharynx part de l'orifice oral ; il s'amincit d'abord pour s'élargir ensuite au niveau de l'extrémité antérieure du macronucléus. Les fibrilles logées dans la paroi du pharynx traversent le corps jusqu'aux " caudalia " et entrent même dans la queue. La paroi du pharynx s'interrompt sur le côté gauche après le second élargissement et il y a une communication directe entre la cavité du pharynx et l'endoplasme.

Le tube anal, prenant naissance dans l'endoplasme du côté ventral, s'ouvre en dehors par un orifice anal derrière la base du faisceau caudal ventral. 
Le macronucléus, de forme allongée, légèrement courbé dans la direction ventrale, se trouve du côté dorsal dans l'ectoplasme un peu à gauche de la ligne dorso-médiane. L'extrémité antérieure du macronucléus touche l'extrémité antérieure du corps, l'extrémité postérieure atteint le faisceau caudal.

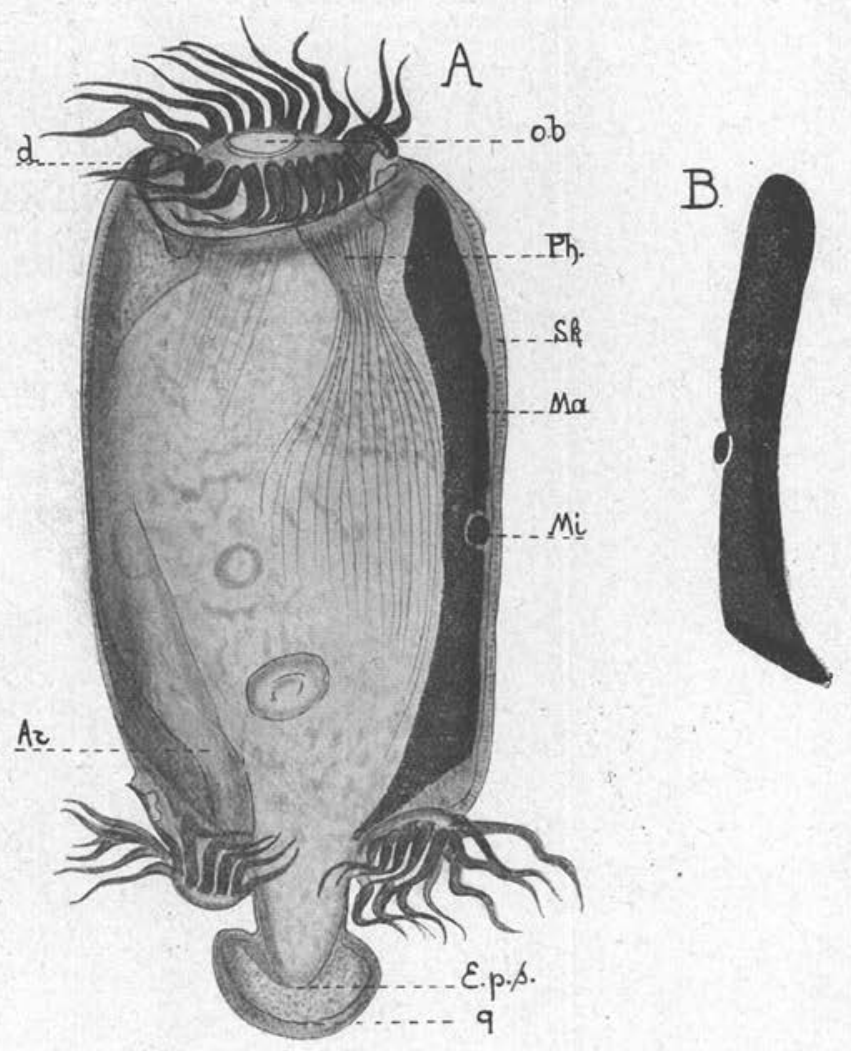

Fıg. 2. - Cycloposthium dentiferum. A. péristome épanoui vu du côté gauche; $\mathrm{B}$, macronucléus vu du côté dorsal ; $\times 280$.

Ce qui attire l'attention, e'est l'absence d'une courbure vers le côté gauche que Gassovsky mentionne dans la description de l'extrémité antérieure du macronucléus chez le $C$. dentiferum. II est inconcevable que, dans sa description, cet auteur indique que l'extrémité du macronucléus se recourbe à gauche, tandis que chez tous les autres Cycloposthium le crochet se recourbe à droite. Je considère que ce qu'il y a de caractéristique chez le $C$. dentiferum. c'est précisément l'absence de crochet à l'extrémité antérieure du 
macronucléus (fig. 2, A). L'extrémité antérieure du macronucléus s'élargit mais reste ou simplement arrondie (après la division ?), ou tronquée. L'extrémité postérieure du macronucléus est aussi un peu élargie et obliquement tronquée dans une direction parallèle à la base du faisceau caudal dorsal. La structure du macronucléus est finement granuleuse. Le micronucléus, de forme éllipsoïdale

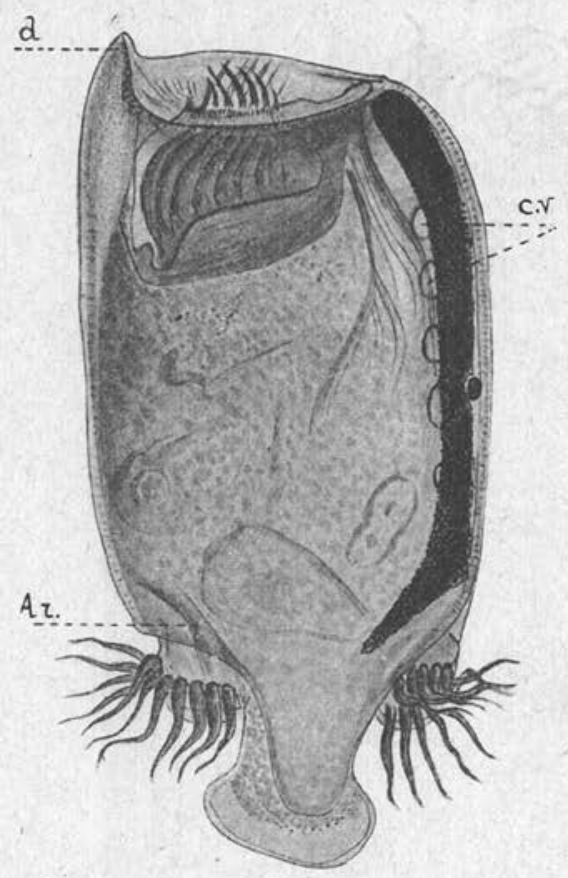

Fıg. 3. - Cycloposthium dentiferum. Péristome invaginé vu du côté gauche $; \times 280$.

$(6 \times 4 \mu)$, est logé dans l'échancrure du macronucléus, à peu près vers son milieu, un peu à gauche de la ligne médio-dorsale.

Les vacuoles contractiles sont situées dans l'ectoplasme, près du macronucléus et sont souvent à peine visibles sur le fond foncé du noyau; leur nombre est de 5 à 6 .

La longueur du corps varie entre 122 et $180 \mu$ (moyenne $150 \mu$ ); la largeur de 60 à $90 \mu$ (moyenne $74 \mu$ ), tandis que l'épaisseur moyenne du corps est à peu près de $50 \mu$. La longueur du macronucléus varie entre 70 et $129 \mu$ (en moyenne $100 \mu$ ).

$\mathrm{Si}$ le $C$. dentiferum est présent, il se rencontre en grande quantité.

En somme, cette espèce présente les particularités suivantes : une excroissance dentiforme au côté ventral de l'extrémité anté- 
rieure, un macronucléus sans crochet à l'extrémité antérieure, un micronucléus situé au niveau de la partie moyenne du macronucléus et une queue droite, un peu élargie à son extrémité. Cette description ne concorde pas avec celle de Gassovsky, mais, je le répète, je considère que cet auteur a confondu les caractères de deux espèces différentes de $C$. dentiferum d'une part et de $C$. edentatum d'autre part, dont nous allons donner la description.

\section{Cycloposthium edentatum n. sp.}

Le corps comprimé latéralement s'amincit un peu vers l'extrémité postérieure ; l'extrémité antérieure du corps est unie, un peu recourbée (fig. 4 C.), le côté dorsal un peu convexe, le côté ventral plus plat. Derrière les " caudalia " le corps s'amincit, formant une queue qui s'élargit tout à coup et forme un crochet ou un fer-à-cheval à deux extrémités dirigées en avant, comme le décrit Gassovsky chez le $C$. dentiferum. D'ailleurs, la plupart des caractères de cette espèce sont ceux du $C$. dentiferum.

Le squelette s'interrompt sur le côté gauche non loin du macronucléus en formant un sillon. Lẻs bords internes des lames de la cuirasse sont unis par un ruban squelettique ("Leiste " de Bundle). Les mêmes relations se retrouvent chez le $C$. dentiferum, mais elles y sont moins nettes sur des préparations totales, car ce ruban squelettique est situé près du macronucléus même. Le long de cette "Leiste ", mais plus ventralement, se trouve dans l'ectoplasme une rangée longitudinale de vacuoles contractiles au nombre de 5 ou 6 . Il y en a 4 immédiatement après la division et 7 avant la division. Les vacuoles s'ouvrent en dehors par des pores bien visibles. La rangée des vacuoles contractiles est dans ce cas à une distance considérable du macronucléus, contrairement à ce qui existe chez le $G$. dentiferum où la rangée de vacuoles est située très près du macronucléus.

Le macronucléus, de forme allongée, a comme toujours une position dorso-latérale. Il y a à l'extrémité antérieure du noyau un crochet recourbé à droite et atteignant $1 / 7$ ou $1 / 4$ de la longueur du macronucléus même (fig. 4 B.). Ce dernier est légèrement recourbé du côté ventral et à droite. Son extrémité postérieure un peu élargie, s'effile vers son extrémité, se recourbant légèrement du côté ventral, le long de la base du faisceau caudal dorsal. Le micronucléus, de forme ellipsoïdale $(7 \times 5 \mu)$, est situé dans l'échancrure du macronucléus, à peu près à une distance de $1 / 3$ de l'extrémité antérieure.

La longueur du corps varie de 130 à $190 \mu$ (moyenne $164 \mu$ ) ; la 
largeur du corps de 60 à $100 \mu$ (moyenne $72 \mu$ ), l'épaisseur moyenne du corps étant à peu près de $50 \mu$. La longueur du macronucléus varie de 66 à $125 \mu$ (moyenne 94 ).

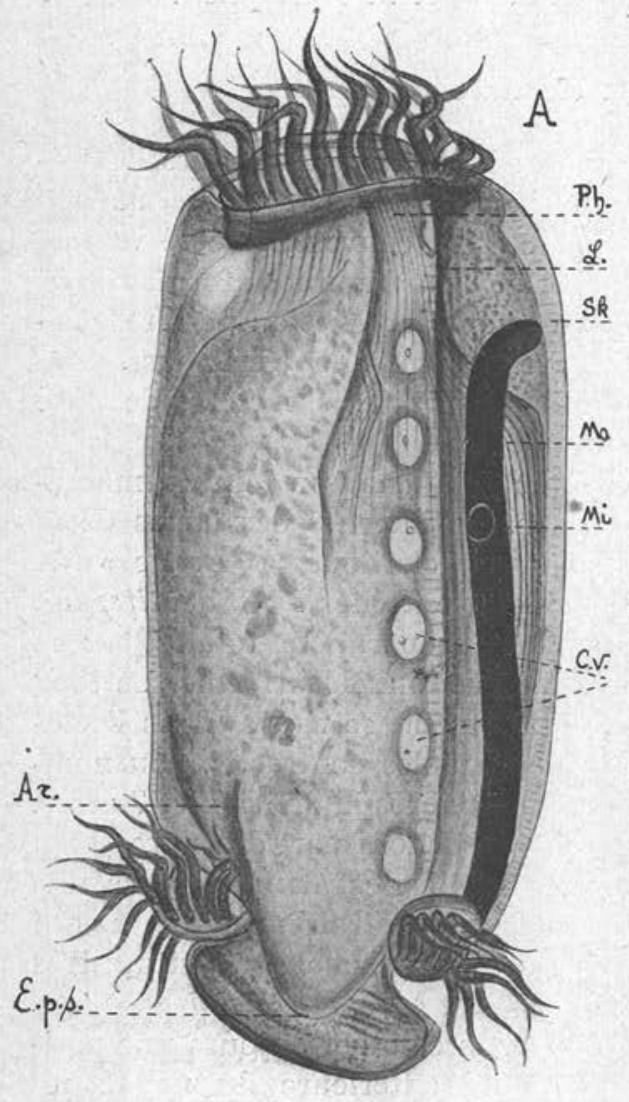

B

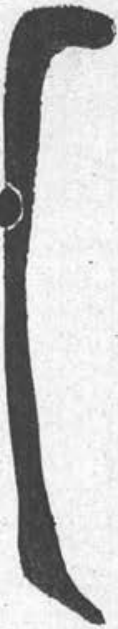

C.

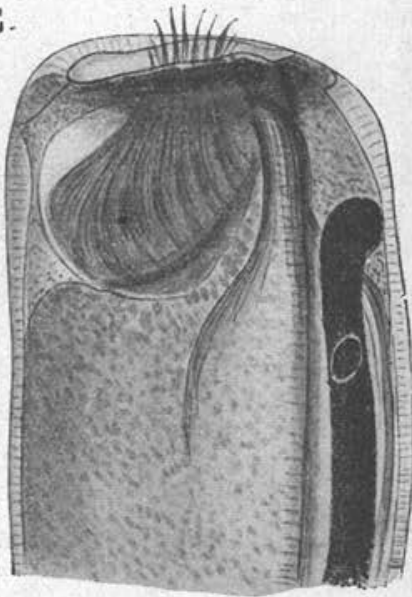

FIG. 4. - Cycloposthium edentatum. A, péristome épanoui vu du côté gauche; $\mathrm{B}$, macronucléus vu du eôté dorsal; C. pôle antérieur avec péristome invaginé vu du côté gauche; $\times 280$.

Cette espèce se rencontre en grand nombre aussi bien associée au C. dentiferum qu'isolément.

Le mouvement des infusoires pendant l'examen sur la table 
chauffante est très rapide et accompagné d'un mouvement giratoire autour d'un axe longitudinal, les membranelles de la zone adoralle y jouant le rôle principal, tandis que les " caudalia " servent en sorte de gouvernail à diriger le mouvement.

Telles, sont les particularités du $C$. edentatum, particularités qui le distinguent du $C$. dentiferum : absence de la dent à l'extrémité antérieure du corps, présence d'une queue fort recourbée, présence d'un crochet à l'extrémité antérieure du macronucléưs, position du micronucléus à une distance de $1 / 3$ de l'extrémité antérieure du macronucléus, enfin le fait que les vacuoles contractiles se trouvent à une certaine distance du macronucléus.

Outre ces traits caractéristiques, ces deux espèces se distinguent encore l'une de l'autre par leurs dimensions et la corrélation des parties. L'analyse biométrique montre clairement cette différence.

Etant à peu près égales en largeur (la différence entre les moyennes pour les deux espèces ne surpasse presque pas l'erreur moyenne de différence), elles sont différentes en longueur.

Par conséquent, on peut prendre comme caractère distinctif le rapport de la largeur du corps à sa longueur, ainsi que le montre le tableau ci-dessous. En outre, le rapport, de la longueur de la queue à la longueur du tronc (sans la queue) montre une autre différence entre les deux espèces (voir le tableau).

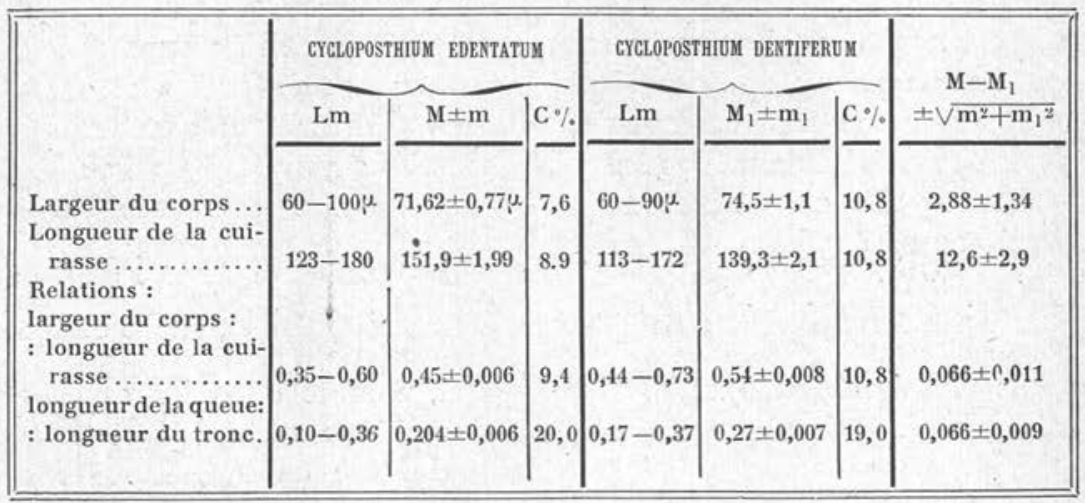

En calculant le rapport de la largeur du corps à sa longueur, j'ai pris au lieu de la longueur du corps la longueur de la cuirasse de forme invariable. J'ai mesuré la longueur du tronc depuis l'extrémité antérieure jusqu'au niveau des bords inférieurs de la base des caudalia, et la longueur de la queue depuis ce niveau jusqu'à l'extrémité de la queue.

Cinquantes mensurations ont ainsi été faites $(n=50)$. 


\section{Cycloposthium piscicauda n. sp.}

Cette espèce m'a été indiquée par le professeur Dogiel dans le matériel qu'il m'a communiqué.

Le corps comprimé latéralement et de forme rectangulaire, s'amincit au niveau des " caudalia ". Le côté ventral est plus plat que le côté dorsal. La forme du corps est stable grâce à la présence de la cuirasse. Les " caudalia " sont situés asymétriquement et la
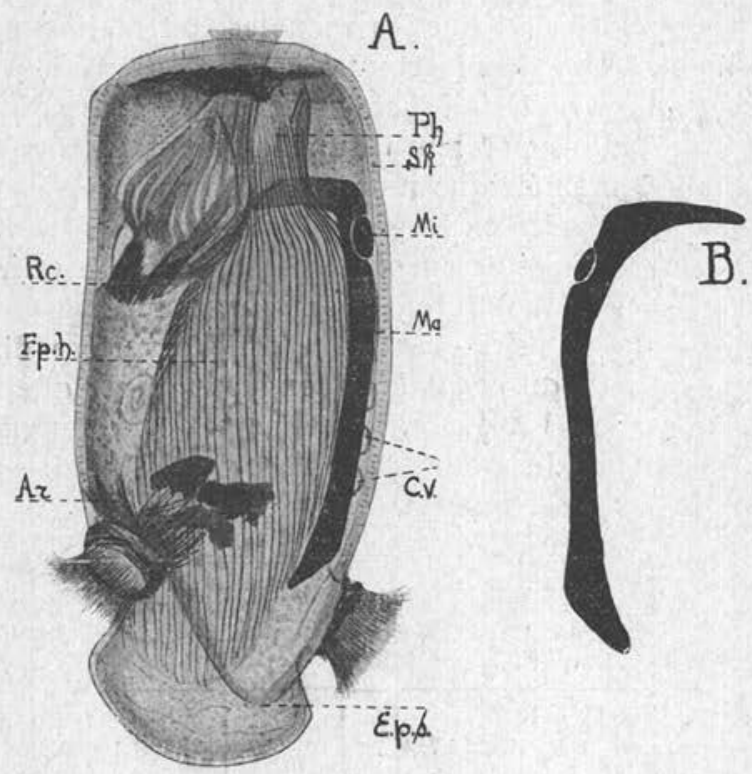

Fıg. 5. - Cycloposthium piscicauda. A, péristome invaginé vu du côté gauche ; B. macronucléus vu du côté dorsal ; $\times 280$.

queue ne s'amincit pas derrière ceux-ci, comme dans les espèces précédentes, mais s'élargit un peu, formant une sorte de queue de poisson, ce qui a contribué à la dénomination de l'espèce. Deux lames squelettiques latérales pénètrent dans la queue.

L'extrémité antérieure du corps est tronquẻe. Deux rétracteurs, invaginant le péristome, sont nettement visibles; ils se laissent bien colorer à l'hématoxyline ferrique et adhèrent au flanc droit du corps (fig. $5 \mathrm{~A}$. et $6 R$. c.).

Le pharynx a la même forme que chez les espèces précédentes. Outre les fibrilles pharyngiennes, on remarque des fibrilles s'éloignant de la base des "caudalia " en dedans du corps et des fibrilles circulaires dans les parois du tube anal. 
Le long de la paroi dorsale du eorps, plus près du côté gauche, dans l'ectoplasme, se trouve le macronucléus d'une forme allongée. légèrement recourbé dans la direction ventrale et à droite. Son pôle antérieur est recourbé en forme de crochet vers le côté droit. Ge crochet est si long, qu'il embrasse la partie amincie du pharynx du côté droit. Les dimensions du crochet atteignent $1 / 4$ ou la moi-

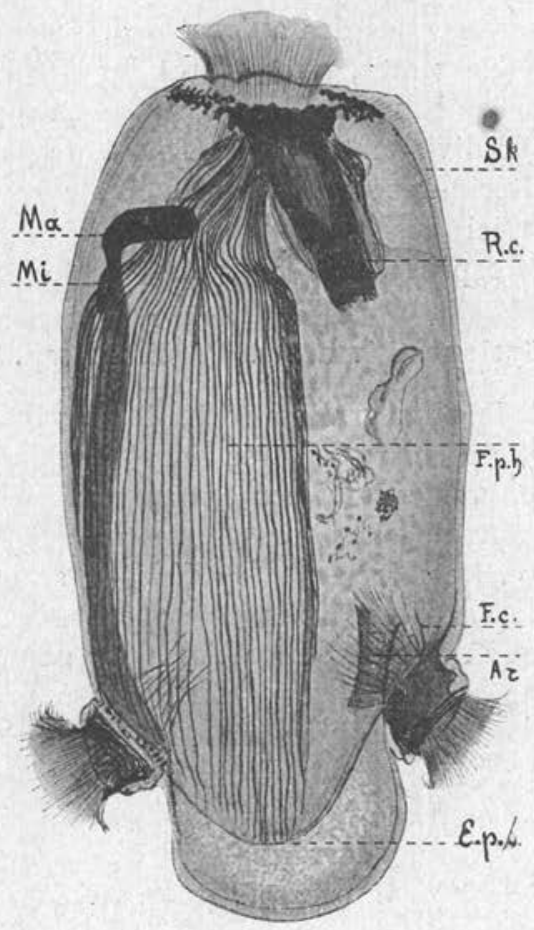

FIG. 6. - Cycloposthium piscicauda, vu du côté droit $; \times 280$.

tié de la longueur du macronucléus (crochet exclu) (fig. 5 B.). L'extrémité postérieure du macronucléus s'élargit un peu et est obliquement tronquée dans une direction parallèle à la base du faisceau caudal dorsal. Le mieronucléus, de forme ellipsoïdale $(6 \times 2,5 \mu)$, est situé près de l'endroit où le macronucléus se courbe pour former le crochet.

Les vacuoles contractiles, au nombre de $4 \grave{a} 5$, sont situées sur le côté gauche du corps aussi près du macronucléus, que, sur les préparations où les infusoires sont surtout couchés sur le flanc; elles ne sont pas visibles, étant cachées par le macronucléus. 
La longueur du corps varie de 125 à $190 \mu$ (moyenṇe $152 \mu$ ); la largeur du corps est de 44 à $80 \mu$ (moyenne $65 \mu$ ).

Cette forme n'a été rencontrée que dans une seule population, associée avec le $C$. bipalmatum.

En somme les particularités de cette espèce sont les suivantes : présence d'une queue en forme de queue de poisson, macronucléus avec un crochet allongé, position du macronucléus et dimensions du corps inférieures à celles des autres formes décrites.

Il est nécessaire de noter, que toutes les formes mentionnées ci-dessus sont proches l'une de l'autre sous le rapport' de la' systématique et que leur division en espèces peut ne pas être définitive. Les recherches ultérieures vérifieront la justesse de mes conclusions, mais jusqu'ici ces formes de grandes dimensions nous paraissent être des espèces bien distinctes.

\section{Cycloposthium scutigerum n. sp.}

Cette espèce est la plus grande de toutes les espèces du genre Cycloposthium; elle a été rencontrée dans plusieurs populations.

Le corps est comprimé latéralement et s'amincit légèrement en arrière. La face ventrale est plate, tandis que la face dorsale est légèrement convexe. Derrière les " caudalia " se trouve une queue recourbée. Le trait caractéristique de cette espèce consiste en un bouclier squelettique spécial, ajusté au côté gauche du corps et s'étendant de l'extrémité antérieure du corps à peu près jusqu'au niveau des " caudalia ". Le bouclier est uni au corps sur presque toute son étendue au moyen d'une mince et iongue côte ectoplasmique (fig. 7 A.). L surface du bouclier est presque plate ou à peine convexe, sa forme est allongée, ellipsoïdale, pointue aux deux pôles. La surface du bouclier est presque parallèle à celles du corps, s'éloignant un peu de ce dernier vers le pôle postérieur. L'épaisseur du bouclier (y compris sa côte basale ectoplasmique). peut être évaluée à la moitié ou au tiers de l'épaisseur du corps. Le bord dorsal du bouclier (fig. 8, B. d.) est longé du côté du dos par une petite crête longitudinale (fig. $8, c$. r.), de sorte qu'il existe entre eux un certain espace en forme de gouttière qui peut devenir nul par suite du rapprochement de la partie dorsale du bouclier et de la crête (fig. 7, A., B., et $8, g$.).

Le côté basal ectoplasmique du bouclier contient une rangée de 5 à 6 vacuoles contractiles s'ouvrant par des pores sur la surface libre du bouclier.

Tout le corps est couvert d'une cuirasse squelettique, particulièrement développée sur la côte ectoplasmique et le bouclier. Sur toute la surface du corps et surtout sur le bouclier, se voit un des- 
sin polygonal, formé, ainsi que je l'ai déjà mentionné, par des alvéoles squelettiques visibles à travers la cuticule.

Le macronucléus allongé, se courbe en crochet à droite à son extrémité antérieure et longe le côté dorsal du corps. Le crochet
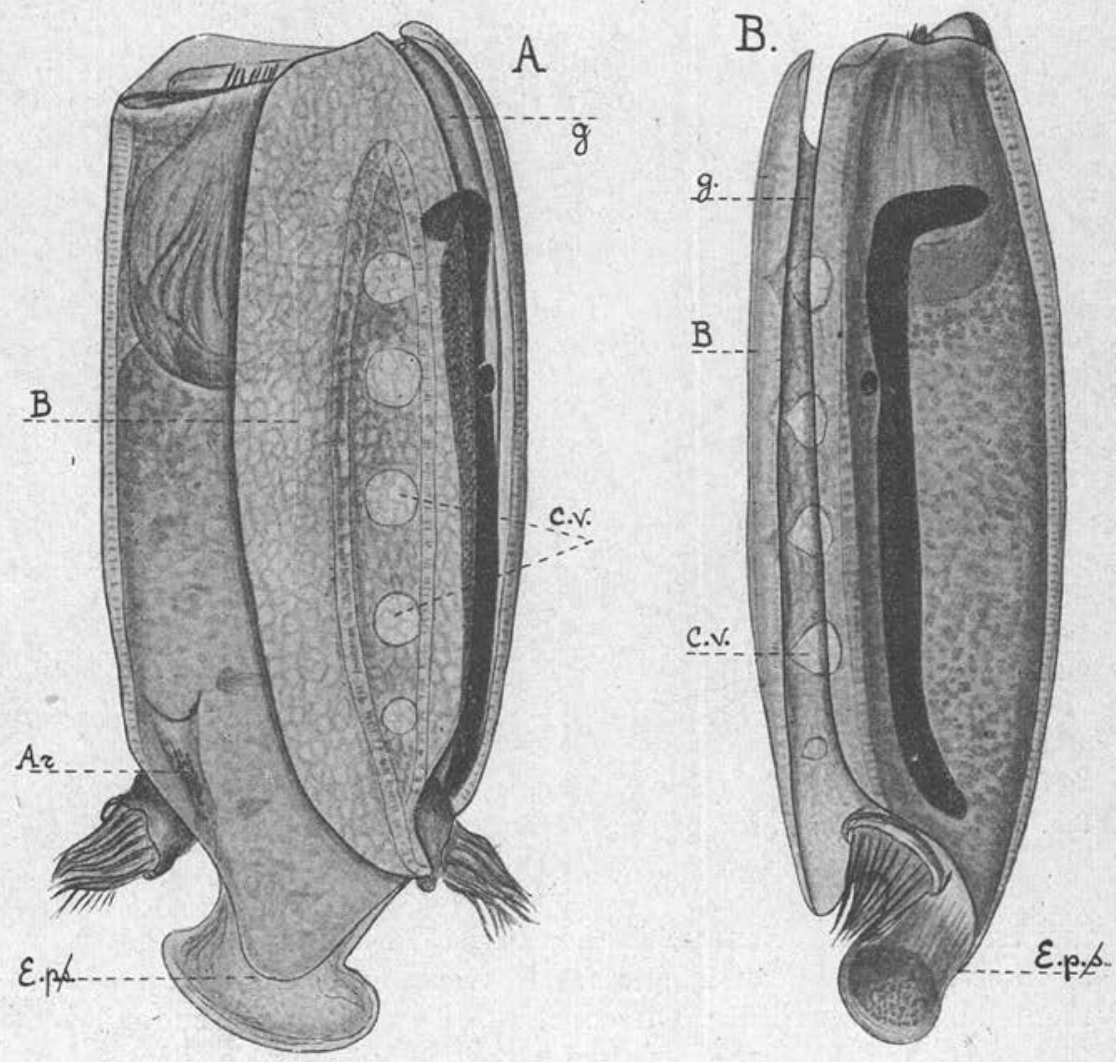

Fig. 7. - Cycloposthium scutigerum. A, vu du côté gauche; B, le même individu vu du côté dorsal ; $\times 280$.

atteint $1 / 5$ ou $1 / 4$ de la longueur du macronucléus entier. A son extrémité postérieure, le macronucléus se courbe un peu vers la partie ventrale.

Le micronucléus, de forme ellipsoïdale $(8 \times 4 \mu)$, est situé dans l'échancrure du macronucléus à une distance d'à peu près un tiers de l'extrémité antérieure de ce dernier.

La longueur du corps varie entre 162 et $290 \mu$ (moyenne $230 \mu$ ); la largeur du corps est de 70 à $160 \mu$ (moyenne $106 \mu$ ); l'épaisseur

AnNales de Parasitologie, T, VI, No $2,-1^{\text {er }}$ avril 1928.

12. 
du corps (sans le bouclier) est de 52 à $90 \mu$. Le rapport de l'épaisseur du corps à la hauteur de la côte ectoplasmique avec le bouclier varie entre 1,1 et 6,4 , le plus souvent $2,4-3,3$ (en moyenne, à peu près 3 ). La longueur du macronucléus est de 84 à $173 \mu$, le plus souvent de 110 à $140 \mu$.

A l'examen des infusoires sur une table chauffante, on constate des mouvements rapides, rectilignes, s'accompagnant d'une rotation autour d'un axe longitudinal. Les infusoires sont très sensibles

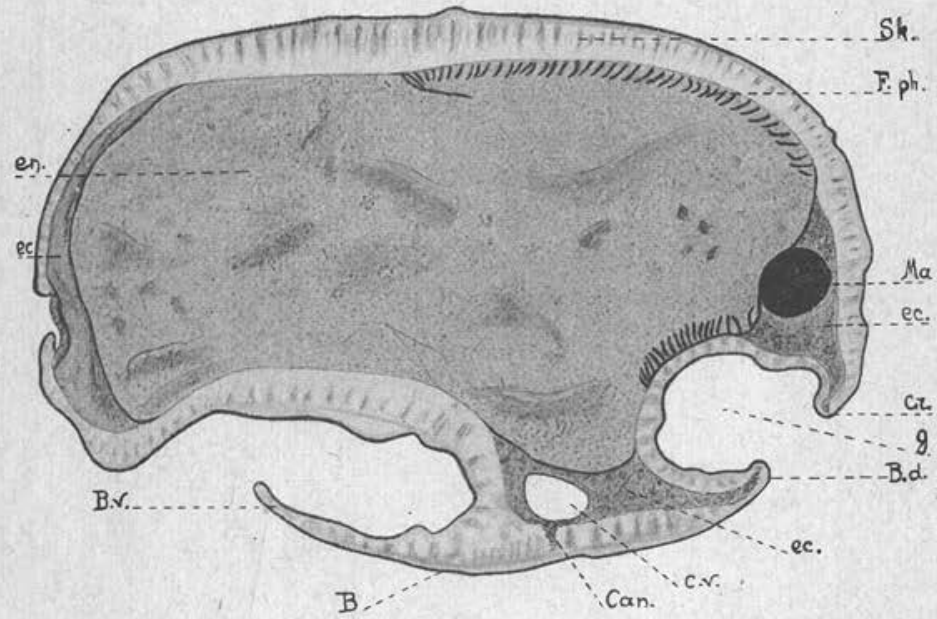

Fıg. 8. - Cycloposthium sculigerum. Coupe transversale au niveau de la troisième vacuole contractile $; \times 560$.

aux irritations extérieures, ainsi le péristome s'invagine et cesse de se mouvoir, quand on frappe sur le verre ou au moindre abaissement de la température. On constate en outre dans l'endoplasme un mouvement distinct des inclusions, ayant lieu de l'extrémité antérieure à l'extrémité postérieure le long du côté dorsal et un mouvement inverse de la queue à la bouche du côté ventral.

La nourriture consiste en matières végétales que l'infusoire avale quelquefois en si grande quantité, que la queue se déforme considérablement. Le mouvement progressif dépend principalement du mouvement des membranelles de la zone adorale.

En terminant, je tiens à exprimer ma profonde reconnaissance à mon maître, le professeur V. Dogiel, pour les précieux conseils qu'il m'a donnés et qui m'ont permis de mener à ìien ce travail.

Laboratoire de Zoologie des inverlébrés à l'Institut des sciences naturelles de Pelerhoff. 